\title{
MULTIPHYSICS MODELLING OF A HYBRID ROCKET ENGINE
}

\author{
Andrea Ferrero', Filippo Masseni², Luca Muscarà ${ }^{3}$ and Dario Pastrone ${ }^{4}$ \\ ${ }^{1}$ Politecnico di Torino, Corso Duca degli Abruzzi 24, 10129 Torino, Italy, andrea_ferrero@polito.it \\ ${ }^{2}$ Politecnico di Torino, Corso Duca degli Abruzzi 24, 10129 Torino, Italy, filippo.masseni@polito.it \\ ${ }^{3}$ Politecnico di Torino, Corso Duca degli Abruzzi 24, 10129 Torino, Italy, s253684@studenti.polito.it \\ ${ }^{4}$ Politecnico di Torino, Corso Duca degli Abruzzi 24, 10129 Torino, Italy, dario.pastrone@polito.it
}

Key words: Hybrid Rocket Engine, Instability, Quasi-1D model, DC shift, Thermal lag

\section{INTRODUCTION}

Hybrid rocket engines (HREs) present interesting advantages over liquid rocket engines (LREs) and solid rocket motors (SRMs). In order to appreciate these advantages, one should look into the different combustion characteristics; in the hybrid engines the combustion occurs in a macrodiffusion flame and the oxidizer to fuel ratio changes along the combustion chamber. In solid rockets the oxidizer and fuel are mechanically or chemically bound in a single solid phase and they burn with a microdiffusion flame while, in the liquid engines, the combustion results from a premixed flame. Thus, unlike hybrids, both these engines have an uniform mixture ratio. On the other hand, in hybrid engines it is possible to throttle by modulating only the liquid flow rate, which is simpler than in a liquid engine where two flow rates must be synchronized.

Furthermore, the European Union is pushing to proscribe some dangerous liquid propellants such as the hydrazine derivatives ${ }^{1}$. As a consequence, there is a huge interest for the "green" propellants and also in this case the HREs present an optimum choice since they employ low toxicity propellants. Indeed, most hybrid propellants and additives are essentially nontoxic, resulting in minimal local environmental impact.

The physical separation of fuel and oxidizer serves also to reduce the probability of an accident, which could lead to propellant release in the environment.

An interesting feature is that the HREs seem viable for the lift-off from Mars because the typical solid fuels employed in HREs, contrary from the ones used for SRMs, do not develop cracks when subjected to wide temperature ranges [1].

From an economical point of view the operational cost for hybrid systems is affordable thanks to their safety features and inert propellant [2].

Despite the several advantages of hybrid systems compared to liquid and solid systems, the hybrids have not seen yet a mass production unlike heritage propulsion systems. In fact, the

\footnotetext{
${ }^{1}$ Pultarova, T., Hydrazine ban could cost Europe's space industry billions, Spacenews, October 25, 2017, viewed December 20, 2020.

https://spacenews.com/hydrazine-ban-could-cost-europes-space-industry-billions/.
} 
HREs suffer from some disadvantages [3]; the most relevant are shown below:

- Low regression rate: the regression rate is the main parameter that brings together HREs and the SRMs. However, HREs have a lower regression rate and this influences the desired thrust level.

- Slow transient: during the course of operation, HREs undergo a number of transients phenomena such as ignition or thrust response to throttling. These effects can be quite important because of the thermal lag in the solid fuel. Furthermore, instability phenomena can be observed and will be investigated in this work.

The transient events have to be analysed to accomplish the thrust requirements of the mission. These events can be classified in four principal phases that are: ignition, throttling, thrust termination and combustion instability.

The last transient phenomenon is the argument of this work. The instability is defined as "the operation condition when the chamber pressure oscillates in a recognizably coherent form with an amplitude of at least 5\% of its mean value 6 [2]

Several theories for the study of instability in LREs and SRMs were developed but they can not be used in HREs. The reason lies in the regression rate law; in the SRMs the regression rate is proportional to the chamber pressure, while in the HREs it is proportional to the oxidizer mass flow. It was seen that the instability is in the form of limited cycle oscillations in the range of low frequencies much smaller than the first longitudinal acoustic mode of the combustion chamber. Moreover, this pressure oscillation could yield to overcome the structural loading or thermal loading. Because of this, it is of paran
this instability.
In this work it was developed a numerica
includes three different sub-models linked tog
thermal sub-model and the chemical sub-mode

The thermal sub-nodel explains the delay
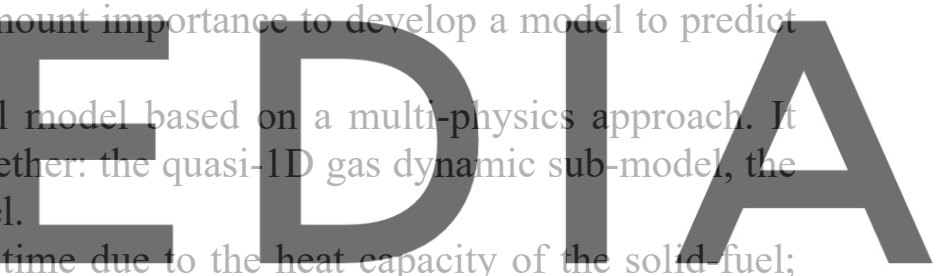

because of this the regression rate can not modify instantaneously to changes in wall heat flux.

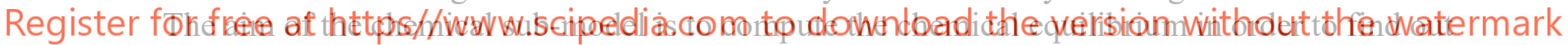

the temperature and the pressure inside the combustion chamber; it also gives the molar fractions of the product species of the reaction. The thermal sub-model requires the total mass flux and the oxidizer mass flux in the chamber, while the chemical sub-model needs the mixture density, the internal energy and the oxidizer to fuel ratio at each point along the chamber. The Q1D gas dynamic sub-model is able to find out the input parameters for the other two submodels. Furthermore, it is able to predict the fluid dynamic flow field along the axis of the chamber and its unsteady evolution. Finally the numerical model is able to analyse the combustion instability and to predict the so-called "DC-shift".

\section{Q1D GAS DYNAMIC SUB-MODEL}

The quasi-one-dimensional gas dynamic sub-model is based on the Euler equations along the longitudinal axis. The model is quasi-one-dimensional because it takes into consideration the cross section average value and the fluid dynamic variables along the longitudinal direction. Throughout the combustion process, the fuel passes through the solid-phase into the gas phase. Therefore, the fuel mass flux is radially injected inside the combustion chamber and a source mass is added in the Euler equations in order to consider this injection. Moreover a further 
equation is introduced, "the mixture fraction equation", following the approach of Karthikeyan, G. and Shimada T. [4]. This equation is similar to the mass conservation equation, but in addition, there is a new variable that multiplies the density, called "mixture fraction".

$$
\epsilon=\frac{b_{c, m i x}-b_{c, o}}{b_{c, f}-b_{c, o}}
$$

In the equation (1) $\epsilon$ is the mixture fraction and $b_{c, \text { mix }}$ represents the mole numbers of atomic Carbon per unit mass of mixture gas, while $b_{c, f}$ and $b_{c, o}$ represent the same quantity for the fuel and the oxidizer stream. In this hybrid rocket engine model, the oxidizer is gaseous oxygen and it does not include any carbon atoms; as a matter of fact $b_{c, o}$ is equal to zero. After these statements, the following equations were used for the Q1D gas dynamic sub-model.

$$
\begin{gathered}
\frac{\partial A \rho}{\partial t}+\frac{\partial A \rho u}{\partial x}=\dot{m}_{f} l_{p} \\
\frac{\partial A \rho u}{\partial t}+\frac{\partial A\left(\rho u^{2}+p\right)}{\partial x}=p \frac{d A}{d x} \\
\frac{\partial A \rho E}{\partial t}+\frac{\partial A(\rho E+p) u}{\partial x}=\dot{m}_{f} l_{p} h_{w}
\end{gathered}
$$
accuracy in space is obtained by using a classical slope limiter (minmod).

The model deveioped in the present work describes an axial injected HRE, which consists of a combustion chamber and a convergent-divergent nozzle. The length of the nozzle is assumed equal to the length of the chamber. The number of grid points is 50 and they are uniformly spaced both in the chamber and in the nozzle. The boundary conditions are imposed only at the inlet; indeed the flow is supersonic in the divergent part of the nozzle. The subsonic boundary conditions are given in table 1 .

Table 1: Subsonic boundary conditions at the inlet

\begin{tabular}{|cc|}
\hline Parameter & Value given \\
\hline Temperature $\mathbf{T}$ & $300 \mathrm{~K}$ \\
\hline Mass flow rate $\dot{\mathbf{m}}_{\mathbf{o x}}$ & $0.14 \mathrm{~kg} / \mathrm{s}$ \\
\hline
\end{tabular}


The fluxes at the inlet are evaluated by taking the boundary conditions given in the table 1 and the pressure advancing from the first cell with a zeroth-order extrapolation.

\section{THERMAL SUB-MODEL}

The fuel flow rate $\dot{m}_{f}$ that appears in the source term of the Q1D gas dynamic sub-model is computed as the product between the fuel density $\rho_{f}$ and the regression rate $\dot{r}$. The onedimensional unsteady heat conduction equation is used to describe the temperature profile inside the solid-fuel and to give the wall temperature that is required for the evaluation of the regression rate. In this work the thermal sub-model is similar to that implemented in [6], however here it was paired with the Q1D gas dynamic sub-model instead of the zerodimensional combustion chamber.

Some assumptions have to be done and according to [7], they are:

- The thermal penetration thickness is much smaller than the fuel port grain; this enables to solve the problem in a planar coordinate system.

- The thermo-physical properties of the solid-fuel are assumed to be constant with the temperature.

- The reaction and melting of the solid-phase are confined inside the thin layer below the regression surface.

The figure 1 shows the schematic temperature profile inside the gas and in the fuel grain; it shows also the velocity profile and the coordinate system (y) used for solving the heat equation

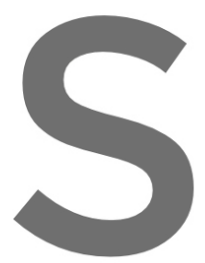

in the solid.
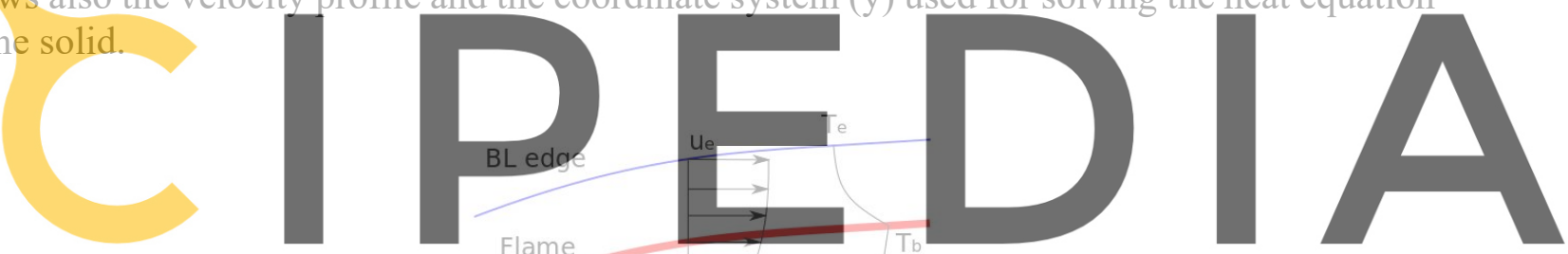

Register for free at https//www.scipedia.com to downnload the version without the watermark

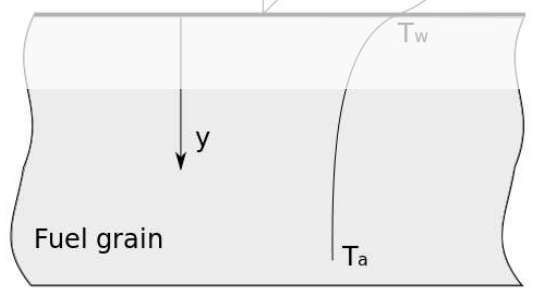

Figure 1: Schematic temperature profile inside the gas and in the fuel-grain.

The one-dimensional unsteady heat conduction equation written in a reference system which follows the moving regression surface is defined as:

$$
\frac{\partial T}{\partial t}=\dot{r} \frac{\partial T}{\partial y}+\alpha \frac{\partial^{2} T}{\partial y^{2}}
$$

where $T, \alpha, \dot{r}, y$ are temperature of the fuel grain, thermal diffusivity, regression rate and radial location. 
The equation (6) is solved once the boundary and the initial conditions are specified. The first boundary condition is the following:

$$
y \rightarrow \infty \quad T=T_{a}
$$

The second boundary condition is imposed at the wall position, and it is:

$$
y=0 \quad \dot{Q}_{w}=-\lambda\left(\frac{\partial T}{\partial y}\right)_{w}+\dot{r} \rho_{f} L_{v}
$$

Where $\dot{Q}_{w}$ is the wall heat flux, $\lambda$ is the thermal conductivity and $L_{v}$ is the latent heat of vaporization.

The initial condition imposes a certain initial temperature distribution inside the solid-fuel.

It is expressed as the following:

$$
t=0 \quad T(y)=T_{i}(y)
$$

\section{A law for the regression rate is necessary to close the problem. An Arrhenius type relation} is used to approximate the pyrolysis or the vaporization process; this relation links the regression rate with the wall temperature as it can be seen below:

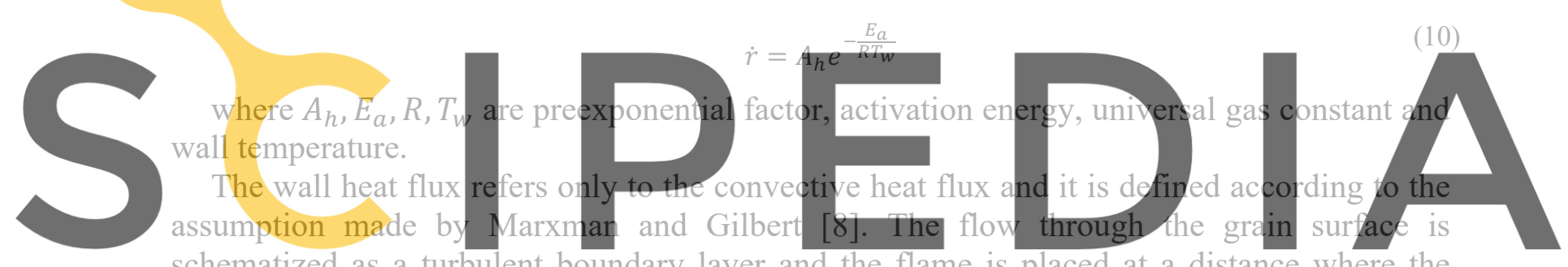

schematized as a turbulent boundary layer and the flame is placed at a distance where the

$$
\dot{Q}_{w}=S t \rho_{b} u_{b} \Delta h
$$

Where $\rho_{b}$ and $u_{b}$ are density and velocity at the flame location while $\Delta h$ is the difference between the enthalpy at the flame and the enthalpy at the wall in the gas phase. Under the assumption that over the whole combustion chamber the turbulent boundary layer is developed, it is possible to link the Stanton number with the skin friction coefficient $C_{f}$ as was done in [9].

$$
S t=\frac{1}{2} C_{f}\left(\frac{\rho_{e} u_{e}^{2}}{\rho_{b} u_{b}^{2}}\right) \Rightarrow \dot{Q}_{w}=\frac{1}{2} C_{f} G\left(\frac{u_{e}}{u_{b}}\right) \Delta h
$$
flux.

Where $\rho_{e}$ and $u_{e}$ are density and velocity at the boundary layer edge and $G$ is the total mass

An important phenomenon is the "blocking-effect"; it is due to the vaporization of the fuel mass. This effect must be considered because it influences directly the wall heat flux and the regression rate. When the regression rate is high, the wall-heat flux decreases due to the blowing of the gas-fuel, but this reduction in the wall-heat flux consequently implies a reduction in the regression rate and the blowing. This is an oscillatory mechanism that affects the transition- 
state during the combustion. In order to take this effect into account, the dimensionless blowing parameter $B$ is used and it is defined as the following:

$$
B=2 \rho_{f} \dot{r} / G C_{f}
$$

Marxman found a relationship between the skin friction coefficient $C_{f}$ and the skin friction coefficient $C_{f 0}$ in the absence of the blowing [10]:

$$
\frac{C_{f}}{C_{f 0}}=\left[\frac{\ln (1+B)}{B}\right]^{0.8}\left[\frac{\left(1+\frac{13 B}{10}+\frac{4 B^{2}}{11}\right)}{(1+B)\left(1+\frac{B}{2}\right)^{2}}\right]^{0.2}
$$

The approach of D. Pastrone and C. Carmicino in [7] was used, instead of the equation (14). Therefore, in the range of $5 \leq B \leq 100$ the equation (14) can be approximated with the following equation.

$$
\frac{C_{f}}{C_{f 0}}=q B^{-k_{b}}
$$

If $B \rightarrow 0$, the equation (15) provides non-physical results, thus, in this case an alternative equation is proposed.

$$
\frac{C_{f}}{C_{f 0}}=\frac{1}{1+0.4 B}
$$

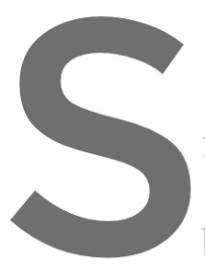

By imposing $q=0$ for $B=B_{\text {ref }}=5.313$

Furthermore, substit blowing parameter are obtained.
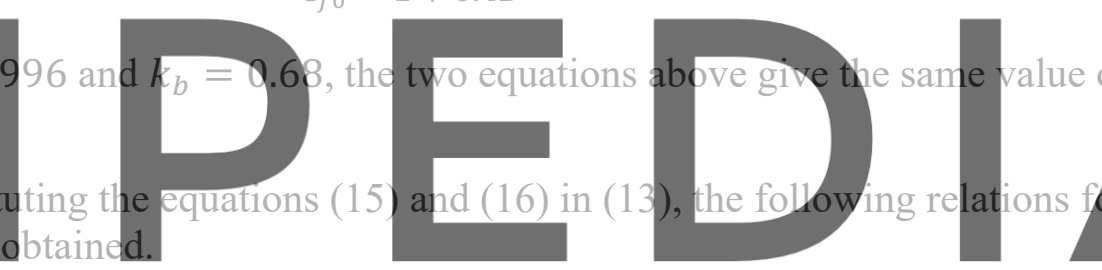

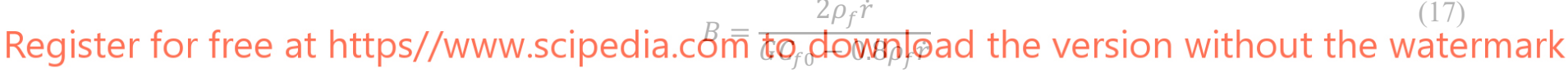

$$
B=\left(\frac{2 \rho_{f} \dot{r}}{q G C_{f 0}}\right)^{\frac{1}{1-k_{b}}}
$$

The wall-heat flux can be rewritten including the thermochemical blowing parameter $B_{t}=$ $\left(\frac{u_{e}}{u_{b}}\right) \Delta h / h_{v}$, where $h_{v}$ is the effective enthalpy of vaporization.

$$
\dot{Q}_{w}=\frac{1}{2} C_{f_{0}} \frac{C_{f}}{C_{f 0}} G B_{t} h_{v}
$$

As a consequence, the wall-heat flux can be written with two equations based on the blowing parameter.

$$
\dot{Q}_{w}=B_{t} h_{v}\left(\frac{1}{2} C_{f 0} G-0.4 \rho_{f} \dot{r}\right) \quad \text { if } B \leq B_{\text {ref }}
$$




$$
\dot{Q}_{w}=\frac{0.996}{2} C_{f 0} G B_{t} h_{v}\left(\frac{2 \rho_{f} \dot{r}}{0.996 G C_{f 0}}\right)^{-\frac{k_{b}}{1-k_{b}}} \text { if } B>B_{\text {ref }}
$$

The quasi-steady wall heat flux can be written by using the reference parameters. As it happens, the relations for the wall heat flux are functions of the oxidizer mass flux $G_{o x}$ and the regression rate as expressed below.

$$
\begin{array}{ll}
\dot{Q}_{w}=A_{1} G_{o x}^{n}-A_{2} \dot{r} & \text { if } B \leq B_{r e f} \\
\dot{Q}_{w}=A_{3} G_{o x}^{\frac{n}{1-k_{b}}} \dot{r}^{-\frac{k_{b}}{1-k_{b}}} & \text { if } B>B_{\text {ref }}
\end{array}
$$

Where the three constant that now appear in the equations are defined as the following.

$$
A_{1}=\frac{1}{2} C_{\text {foref }} G_{\text {oxref }}^{1-n} B_{\text {ref }} h_{\text {vref }} ; \quad A_{2}=0.4 \rho_{f} B_{\text {ref }} h_{\text {vref }} ; \quad A_{3}=\frac{\dot{Q}_{w r e f}}{\left(G_{\text {oxref }}^{n} \dot{r}_{\text {ref }}^{-k_{b}}\right)^{\frac{1}{1-k_{b}}}}
$$

The unsteady heat equation was solved by applying the finite volume method and the forward Euler method. The wall temperature is found at each time step by solving the equation (8) with the Bisection method because of the presence of the wall temperature in the regression rate equation (10).

The domain of the solid-fuel is set $[0,100 \delta]$ where $\delta=\alpha / \dot{r}_{\text {ref }}$ is the thermal thickness in

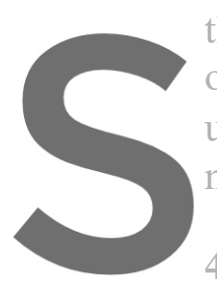
the solid. After a prelin of the large temperature uniformly; the first noder nodes increases by 11 4
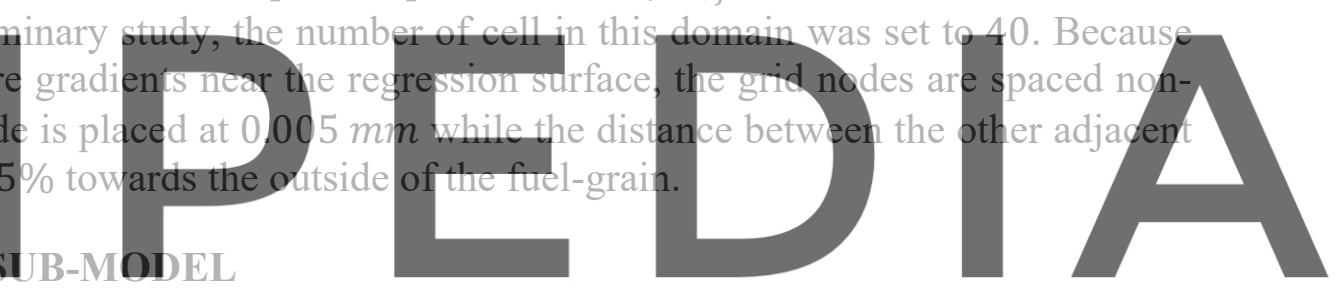

A chemical model is required because of the chemical reaction occurring inside the

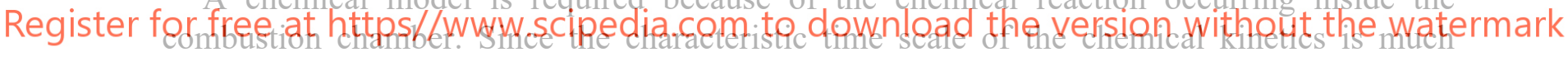
smaller than the fluid dynamic ones, it can be possible to consider the chemical equilibrium model without introducing a significant error.

The numerical code applied to solve the chemical equilibrium is the NASA CEA code [11]; it uses a solution method based on the minimization of free energy. The free energy could be the Gibbs energy or the Helmhotz energy, depending on the thermodynamic state variables chosen as inputs. In our work, we chose the Helmhotz energy because at each time step the density and internal energy of the mixture are known from the Q1D gas dynamic sub-model. Beyond these parameters, the CEA needs the moles of the reactants and they are derived from the mixture fraction by solving the fourth equation of the Q1D gas dynamic sub-model. Once specified the inputs, it gives as results the thermodynamic variable and the mole fractions. In this case, the temperature and the pressure are the variables of interest.

The CEA code also offers the chance to choose the product species. It is good practice to select certain species, the significant ones, so as to simplify the complexity of the problem.

In this work the CEA code is used only to compute the molar fractions: the temperature of the mixture is obtained iteratively by using the Newton's method. 
For each species, the specific enthalpy $H^{\circ}$ and the specific heat at constant pressure $C_{p}{ }^{\circ}$ are functions of the temperature and they have a fourth order polynomial form according to [11].

$$
\begin{gathered}
\frac{H^{\circ}}{R T}=-a_{1} T^{-2}+a_{2} T^{-1} \ln T+a_{3}+a_{4} \frac{T}{2}+a_{5} \frac{T^{2}}{3}+a_{6} \frac{T^{3}}{4}+a_{7} \frac{T^{4}}{5}+\frac{a_{8}}{T} \\
\frac{C_{p}^{\circ}}{R}=a_{1} T^{-2}+a_{2} T^{-1}+a_{3}+a_{4} T+a_{5} T^{2}+a_{6} T^{3}+a_{7} T^{4}
\end{gathered}
$$

In those functions, the constant coefficients $a_{i}$ are stored into the library file. Furthermore, there are three sets of constant coefficients defined for three different temperature ranges.

$$
T_{k+1}=T_{k}-\frac{f\left(T_{k}\right)}{f^{\prime}\left(T_{k}\right)}
$$

In the equation above, the function $f\left(T_{k}\right)$ and its derivate $f^{\prime}\left(T_{k}\right)$ have the following forms:

$$
\begin{aligned}
& f\left(T_{k}\right)=\frac{e}{R}-\frac{H^{\circ}}{R}+\frac{T_{k}}{P M}=0 \\
& f^{\prime}\left(T_{k}\right)=-\frac{C_{p}^{\circ}}{R}+\frac{1}{P M}=0
\end{aligned}
$$

Where $e$ is the specific internal energy and $P M$ is the molecular weight of the mixture.

Once the method converges with a demanded tolerance, the temperature is known and the pressure can be valuate
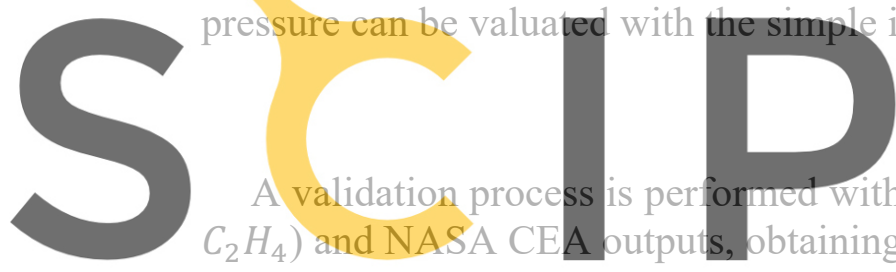

$p=\rho$
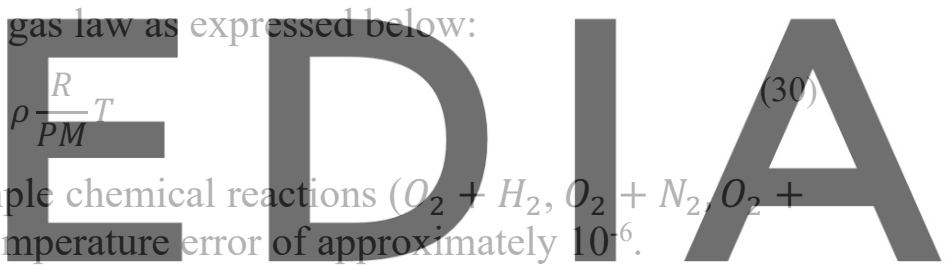

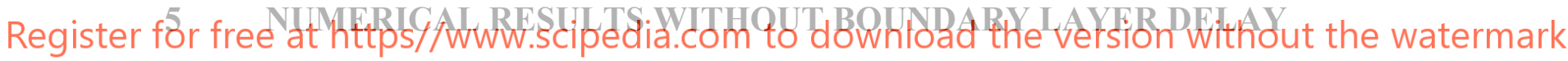

In this work, the propellant combination is HDPE and gaseous oxygen. It was assumed that the pyrolysis process takes place instantaneously and as a consequence gaseous ethylene can be considered as fuel. The geometry of the model is schematized in the figure 2 . Moreover, the radial injection of the fuel starts at the non-dimensional position $x / L=0.1$ and ends at $x / L=$ 0.5 . The convergent-divergent nozzle lies from $x / L=0.5$ to $x / L=1$.

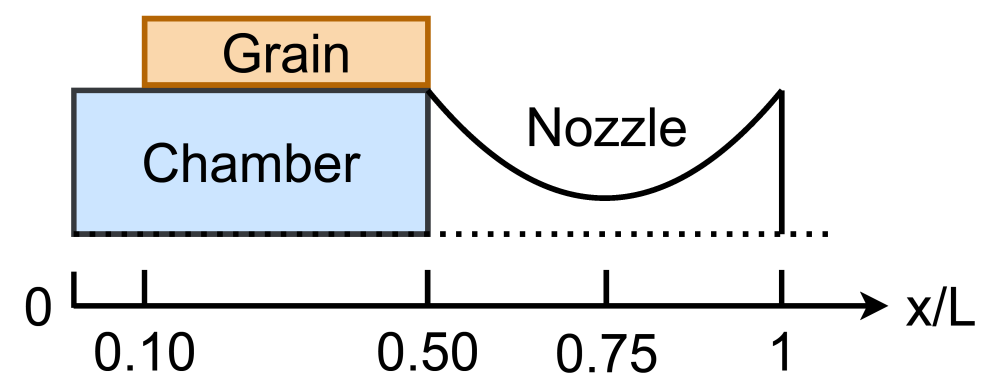

Figure 2: Schematic of combustor and nozzle in the meridional plane. 
A first simulation is performed with the previously defined equations in which the boundary layer delay does not appear. The effects of the boundary layer delay will be introduced in the next Section.

The significant variables, calculated on the axial location through the combustion chamber and the nozzle, are shown in the figures below. Besides, the figures represent the steady solution of the numerical model. The graph on the left of the figure 3 illustrates the fluid dynamic flowfield in terms of Mach number, non-dimensional temperature and non-dimensional pressure. It can be noticed that the temperature rises from $x / L=0.1$ to $x / L=0.5$, according to the radial injection of the fuel, while decreases in the last part of the domain towards the end of the nozzle. As we expected, the Mach number increases in the convergent-divergent part by reaching the unity in the throat of the nozzle.

In the figure 3, the graph on the right, displays the mixture fraction inside the domain. It puts in evidence the effects of the fuel injection; therefore, it is constant from $x / L=0.5$ to $x / L=1$ in line.

The left plot of the figure 4 exhibits the regression rate behaviour and it can be seen that its value rises from 0 to almost $0.6 \mathrm{~mm} / \mathrm{s}$ and then drops to 0 in the nozzle. Finally, the right plot of the figure 4 shows the pressure history in a station in the middle of the chamber: the pressure reaches a steady solution and the oscillations generated by the initial transient disappear.

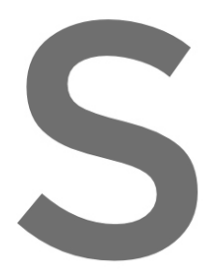

MACH, NON-DIMENSIONAL TEMPERATURE AND PRESSURE THROUGH COMBUSTION CHAMBER AND NOZZLE
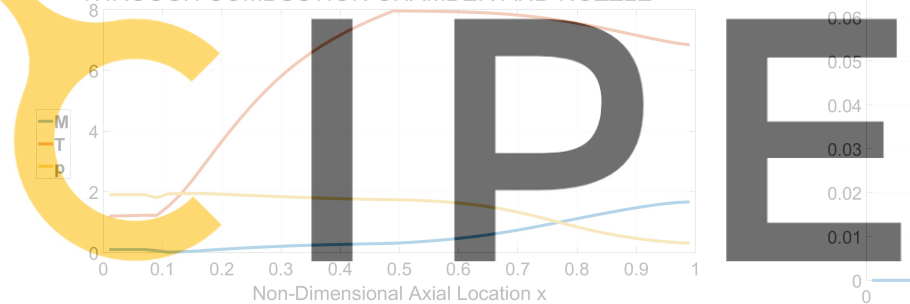
MIXTURE FRACTION,

Register for free at https//www.scipedia.com to download the version without the watermark Figure 3: Steady-state solution: a) Fluid-dynamic flow field, b) Mixture fraction.

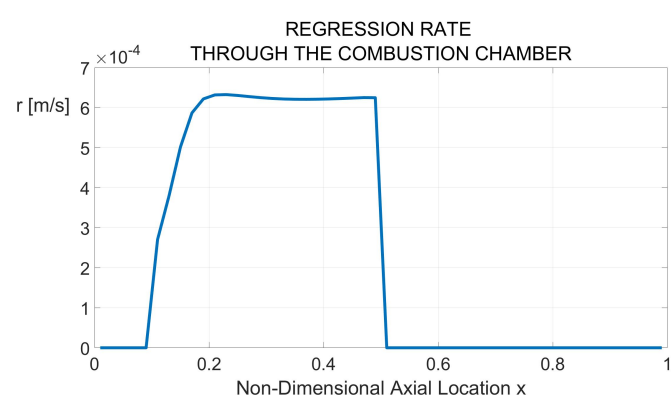

a)

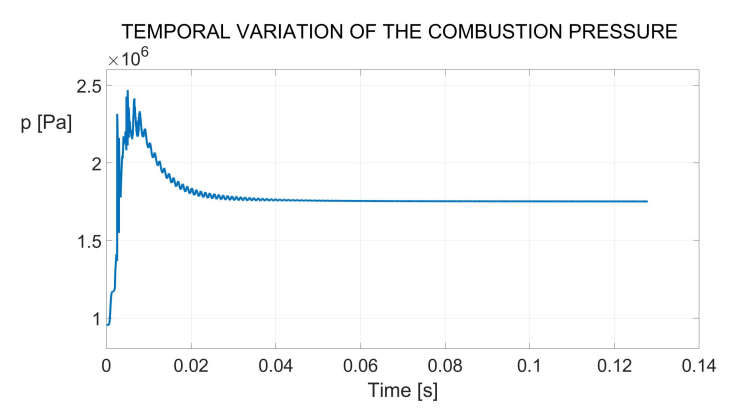

b)

Figure 4: Steady-state solution: a) Regression rate, b) Pressure history in the half chamber. 


\section{6}

NUMERICAL RESULTS WITH THE BOUNDARY LAYER DELAY

During the transient, the convective heat transfer to the surface is related to the regression rate through the blocking effect due to the blowing of the gas. These dynamics together with the thermal lag lead to the instability oscillation.

According to Karabeyoglu et al. in [12], the instability takes place once the blocking effect due to the radial injection of the fuel mass, is coupled together with the thermal transient in the solid and the boundary delay dynamic. Among these physical phenomena, the boundary layer delay is the cause of the primary oscillation frequency of the chamber pressure.

The experimental tests showed a broad peak in the low frequency regime, namely the lowfrequency hybrid instability. So as to analyse the instability behaviour of the chamber pressure the delay time was included between the regression rate and the wall heat flux in the equations (22) and (23).

$$
\begin{array}{ll}
\dot{Q}_{W}=A_{1} G_{o x}^{n}-A_{2} \dot{r}\left(t-\tau_{B L}\right) & \text { if } B \leq B_{\text {ref }} \\
\dot{Q}_{W}=A_{3} G_{o x}^{\frac{n}{1-k_{b}}} \dot{r}\left(t-\tau_{B L}\right)^{-\frac{k_{b}}{1-k_{b}}} & \text { if } B>B_{\text {ref }}
\end{array}
$$

The parametric study was done by varying the boundary layer delay time $\tau_{B L}$. The pressure history in the middle of the combustion chamber was recorded in order to perform the fast Fourier transform and get the spectrum. The frequency spectrum, for different value of $\tau_{B L}$. shows a peak in the ld
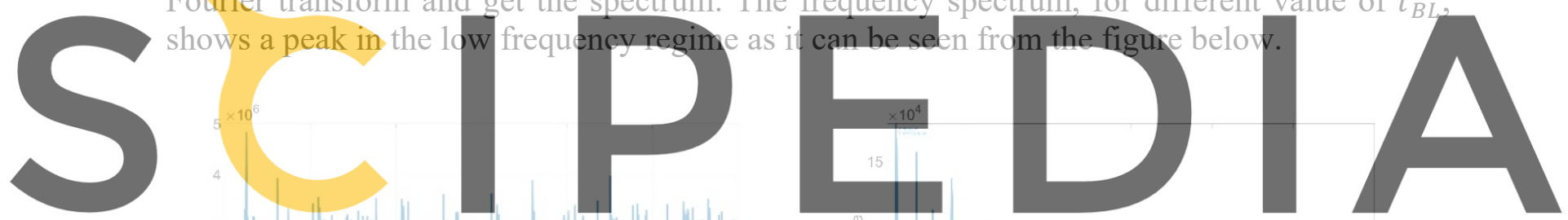

Register for free at http\$//www.scipedia.com to download the version without the watermark
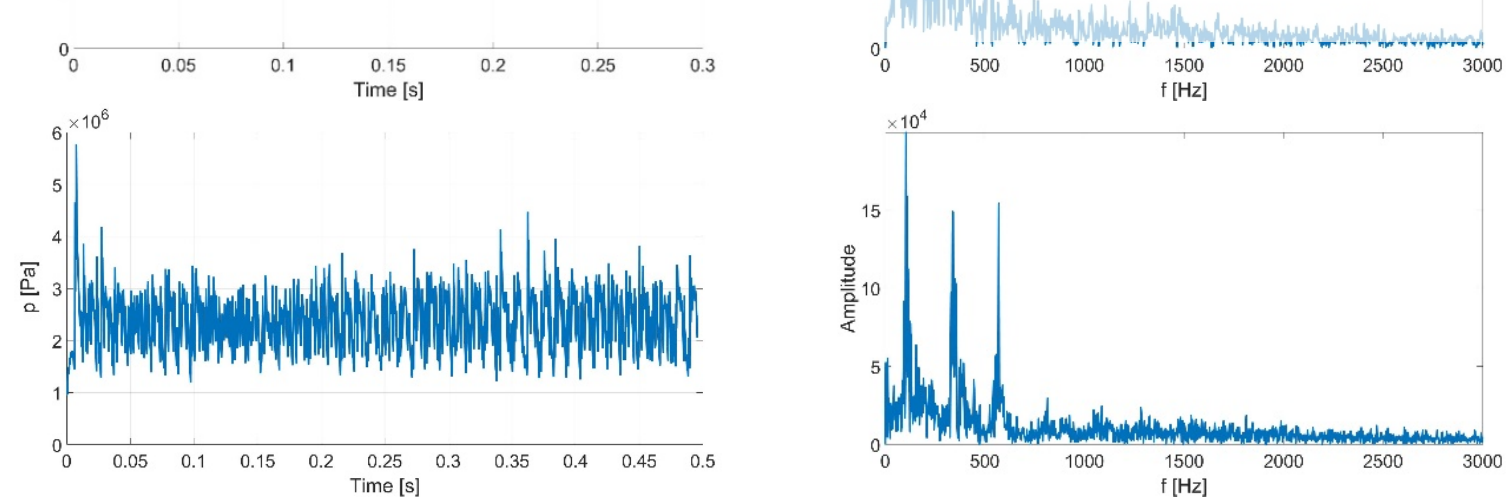

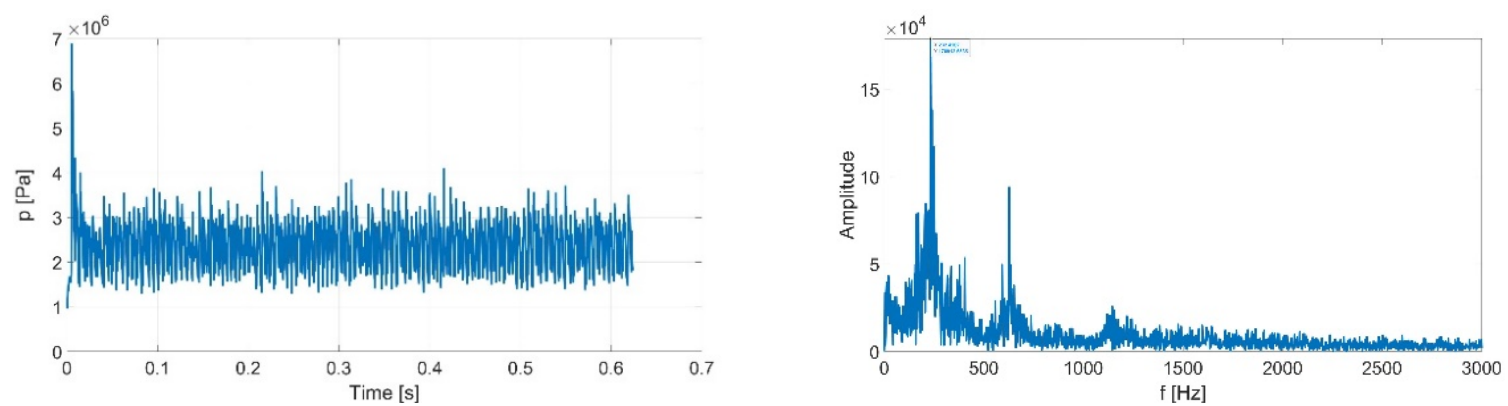

Figure 5: Pressure histories (left side) and spectrum (right side) for $\tau_{B L}=10 \mathrm{~ms}, 5 \mathrm{~ms}$ and $2.5 \mathrm{~ms}$ (from top to bottom).

It is worth noting that the mean values of the pressure histories, observed in the figure above, rise to more than $2 \cdot 10^{6} \mathrm{~Pa}$ against the value of $1.8 \cdot 10^{6} \mathrm{~Pa}$ achieved with $\tau_{B L}$ equal to zero, in the right plot of the figure 4 . This effect is the so-called "DC-shift".

Karabeyoglu et al. [12] suggested a semi-empirical correlation able to quantify the link between the instability frequency and the boundary layer delay time.

$$
f=\frac{0.48}{\tau_{B L}}
$$

The numerical results obtained by the present work are compared with this correlation (33) and it was found that they are in good agreement with the theoretical trend.

\section{CONCLUSIONS}

The numerical model developed in this work seems capable of reproducing the main phenomena which govern the instability and the DC-shift. This is done by taking into account the three fundamental phenomena which are responsible for this behaviour, according to Karabeyoglu et al. [12]: blocking effect, thermal lag and boundary layer delay.

The quasi 1D gas-dynamics model developed in this work allows to estimate the spatial distribution of the unsteady pressure field in the chamber.

A parametric analysis on the boundary layer delay time was performed and the predicted frequency for the first peak in the spectrum is in good agreement with the correlation described by the eq. (33). 


\section{REFERENCES}

[1] Boiron, A. J. and Cantwell, B. J., Hybrid Rocket Propulsion and In-Situ Propellant Production for Future Mars Missions, 49 th AIAA/ASME/SAE/ASEE Joint Propulsion Conference, San Jose, CA, July 14-17, (2013).

[2] Chiaverini, M., J., Fundamentals of Hybrid Rocket Combustion and Propulsion, American Institute of Aeronautics and Astronautics, Vol. 218, (2007).

[3] Pastrone, D., Approaches to Low Fuel Regression Rate in Hybrid Rocket Engines, International Journal of Aerospace Engineering, Hindawi Publishing Corporation, Volume 2012, Article ID 649753, April 5, (2012).

[4] Karthikeyan, G., and Shimada, T., Numerical Parametric Analysis of Combustion Instability in Axial-Injected Hybrid Rocket Motors, Journal of Propulsion and Power, (2018).

[5] LeVeque, R. J., Finite Volume Method for Hyperbolic Problems, Cambridge Texts in Applied Mathematics, Volume 31, in Cambridge University Press, August 26, (2002), pp.232-234.

[6] Barato, F., Bellomo, N., Faenza, M. and Lazzarin, M., Numerical Model to Analyze Transient Behavior and Instabilities on Hybrid Rocket Motors, Journal of Propulsion and Power, Vol. 31, No. 2, March-April (2015).

[7] Pastrone, D., and Carmicino, C., On the Explanation of the "DC Shift" in Hybrid Rockets, AIAA Propulsion and Energy Forum, July 9-11, (2018).

[8] Marxman, G. A. and Gilbert, M., Turbulent boundary layer combustion in the hybrid rocket, Ninth International Symposium on Combustion, Academic Press, New York, (1963), pp. 371-383.

[9] Karabeyoglu, M. A. and Altman, D., Dynamic Modeling of Hybrid Rocket Combustion, Journal of Propulsion and Power, Vol. 15, No. 4, July-August (1999).

[10] Marxman, G. A., Combustion in the turbulent boundary layer on a vaporizing surface, Symposium (International) on Combustion, Volume 10, Issue 1, (1965).

[11] Gordon, S., and McBride, B. J., Computer Program for Calculation of Complex Chemical Equilibrium Compositions and Applications, NASA Reference Publication 1311, October (1994).

[12] Karabeyoglu, M. A., De Zilwa, S., Cantwell, B., Zilliac, G., Modeling of Hybrid Rocket Low Frequency Instabilities, Journal of Propulsion and Power, Vol. 21, No. 6, NovemberDecember (2005). 